\title{
Trends in the use of health services and their relationship with multimorbidity in Brazil, 1998-2013
}

\author{
Ana Sara Semeão de Souza ${ }^{1}$ (1) and José Ueleres Braga ${ }^{2,3^{*}}$ (D)
}

\begin{abstract}
Background: Chronic non-communicable diseases (NCDs) are the leading cause of multimorbidity. Access to effective and equitable health services that meet NCDs' needs is still limited in many countries. This constitutes the main barrier to coping with NCDs, especially in minimising the suffering of those who are already sick. The present study aimed to identify the relationship between multimorbidity and the use of different health services in Brazil from 1998 to 2013.

Methods: This is a panel study using data from the health supplement of the National Household Sample Survey of 1998, 2003 and 2008 and data from the National Health Survey carried out in 2013. Three health service utilization outcomes were considered: 1. search for health services in the last 15 days (excluding dental services), 2. medical consultation in the previous 12 months and 3. hospitalisations over the last 12 months. Multimorbidity was assessed by counting the number of morbidities from a list of 10 morbidities. Poisson regression models stratified by sex were used to estimate the crude and adjusted prevalence ratios and their respective $95 \%$ confidence intervals for each outcome of health service use and multimorbidity, per year.
\end{abstract}

Results: There was an increase in the prevalence of demand for health services and medical consultations in the last 12 months between 1998 and 2013, regardless of the multimorbidity classification. The prevalence of hospitalisations has decreased over the study period and increased twofold in individuals with multimorbidity. Having multimorbidity increased the use of health services for the three outcomes under the study, being more expressive among men.

Conclusions: This study found that individuals with multimorbidity have higher levels of use of health services. Better understand the multimorbidity epidemiology and the associated impacts on the use and costs of health services can increase the quality of care provided to these patients and reduce rising health care costs.

Keywords: Multimorbidity, Health services research, Chronic disease, Multiple chronic conditions

\footnotetext{
* Correspondence: ueleres@gmail.com

${ }^{2}$ Departamento de Epidemiologia, Fundação Oswaldo Cruz, Escola Nacional de Saúde Pública Sergio Arouca, Rio de Janeiro, RJ, Brazil

${ }^{3}$ Departamento de Epidemiologia, Instituto de Medicina Social, Universidade do Estado do Rio de Janeiro, Rio de Janeiro, RJ, Brazil

Full list of author information is available at the end of the article
}

(c) The Author(s). 2020 Open Access This article is licensed under a Creative Commons Attribution 4.0 International License, which permits use, sharing, adaptation, distribution and reproduction in any medium or format, as long as you give appropriate credit to the original author(s) and the source, provide a link to the Creative Commons licence, and indicate if changes were made. The images or other third party material in this article are included in the article's Creative Commons licence, unless indicated otherwise in a credit line to the material. If material is not included in the article's Creative Commons licence and your intended use is not permitted by statutory regulation or exceeds the permitted use, you will need to obtain permission directly from the copyright holder. To view a copy of this licence, visit http://creativecommons.org/licenses/by/4.0/ The Creative Commons Public Domain Dedication waiver (http://creativecommons.org/publicdomain/zero/1.0/) applies to the data made available in this article, unless otherwise stated in a credit line to the data. 


\section{Background}

Chronic non-communicable diseases (NCDs) are the leading cause of morbidity and mortality worldwide and approximately three-quarters of deaths due to NCDs occur in low and middle-income countries [1-3]. A challenge for reducing the disease burden of NCDs, multimorbidity is defined as the coexistence of two or more chronic conditions in the same individual [4]. A recent systematic review estimated the combined global prevalence of multimorbidity to be $33.1 \%$. In high-income countries, the prevalence of multimorbidity was $37.9 \%$ and for low and middle-income countries, 29.7\% [5].

In Brazil, the prevalence of multimorbidity was estimated at $24.2 \%$ in 2013 ; it was more frequent in women, individuals with less education and at older ages [6]. Besides, it was observed that in Brazil, people develop morbidity and multimorbidity at a younger age than people living in richer countries and women, 10 years earlier compared to men [6-8].

Recent studies have shown that multimorbidity is associated with increased disability and functional decline, reduced well-being and quality of life, and disproportionately higher levels of use of health services with high costs out-of-pocket [9-13]. Currently, the main health care model is focused on the disease rather than the person, therefore, the participation of different caregivers in the management of multiple conditions is inevitable and often results in competing treatments, ill coordination, and inefficient communication between patients and providers, and even unnecessary replication of diagnostic tests or treatments [14-16].

According to the World Health Organization, access to effective and equitable health services that meet the needs of people with NCDs is still limited in many countries. This constitutes the main barrier to coping with NCDs, especially in minimizing the suffering of those who are already sick [17]. Regarding the determinants of the use of health services, it is known that health needs or the existence of the disease, as well as severity and urgency, are proximal factors of use [18].

Despite the high prevalence of multimorbidity in Brazil, only few published studies have examined its relationship with health services [19-21], and none of these studies characterized the patterns of use of health services in individuals with multimorbidity amongst the Brazilian population. With the increasing demand for health systems, it becomes more necessary to identify the profile of individuals with multimorbidity who need health care more urgently, thus aiming to allocate these resources in the most efficient way possible. In this sense, the present study aimed to identify the relationship between multimorbidity and the use of different health services in Brazil, according to their characteristics from 1998 to 2013.

\section{Methods}

\section{Study design and population}

This was a panel study using data from the health supplement of the Brazilian National Household Sample Survey (PNAD) of 1998, 2003 and 2008 and data from the Brazilian National Health Survey (PNS) conducted in 2013. These surveys were carried out by the Brazilian Institute Geography and Statistics (IBGE) in collaboration with the Ministry of Health. Both studies were based on a probabilistic sample of households obtained in three stages of selection and are representative of the Brazilian population. In 1998, 2003, 2008 and 2013, 344, $975,384,834,391,868$ and 205,546 people participated in the survey, respectively. For this work, the study population consisted of adults aged 18 years or over, totaling 889,941 people.

\section{Study variables}

All variables in this study were extracted from the databases of national surveys collected with the same question or an equivalent question repeated in all years. Three outcomes for the use of health services were considered: 1. search for health services in the last 15 days (excluding dental services), 2. medical consultation in the last 12 months and 3. hospitalisations in the last 12 months. The search for services and hospitalisations were assessed dichotomously (yes/no).

Medical visits in the last 12 months for the years 1998, 2003 and 2008 were measured using the question: "In the past 12 months, have you seen a doctor?" (yes/no). In 2013, the question used was: "When did you last see a doctor?" and the options were: (i) in the last 12 months, (ii) from 1 year to less than 2 years, (iii) from 2 years to less than 3 years, (iv) 3 years or more, and (v) never went to the doctor. To standardize the responses, the 2013 survey variable was recategorized as 'yes' for those who answered affirmatively for option (i) and 'no' for those who opted for the other responses (ii, iii, iv and v).

Multimorbidity was assessed by counting the number of self-reported morbidities, following the definition most used in the literature, that is, the presence of two or more chronic problems in the same individual [22, 23]. The following morbidities were included in the list: (a) chronic back problem, (b) arthritis or rheumatism, (c) cancer, (d) diabetes, (e) bronchitis or asthma, (f) arterial hypertension, (g) heart disease, (h) chronic kidney disease, (i) depression and (j) tendinitis/tenosynovitis. All morbidities weighted 1.0 (one) in the total morbidity count. From the count, multimorbidity was recognised as the presence of two or more morbidities (yes) and one or no morbidity (no).

The selection of independent variables was based on the behavioral model for the use of health services by 
Andersen (1995) [24], which classifies the variables into predisposing, enabling factors and health needs. Predisposing factors were assessed using the variables gender (female/male), categorized age (18-29, 30-39, 40-49, 50-59, 60-69 and $\geq 70$ years), education (no schooling, incomplete elementary school, complete elementary school, incomplete high school, complete high school, incomplete university and completed university), and race/color (white, brown, black, yellow and indigenous).

The enabling factors were measured through possession of a health plan (yes/no) and registration primary care in family health teams (FHT) (yes/no), for the latter there is information only for the years, 2008 and 2013. Health needs were assessed through self-assessment of health status (very good, good, fair, poor and very bad) and limitation of usual activities in the last 15 days (yes/ no). The questions regarding the study variables according to the surveys are available in the supplementary material (Additional file 1).

\section{Data analysis}

Prevalence was estimated for each outcome of health service use stratified by multimorbidity according to the independent variables, for each year of the panel. The Wald test was used for the linear trend between the outcome and the independent variables. Trends with a $p$-value of less than 0.05 were considered statistically significant. Poisson regression models stratified by sex were used to estimate the crude and adjusted prevalence ratios (PR) and their respective 95\% confidence intervals (95\% CI) for each outcome of health service use and multimorbidity, per year. The initial models were progressively adjusted for predisposing, enabling factors, and health needs. The Stata SE 15.0 application (College Station, TX, USA) was used for statistical analysis, and the sample parameters and weights of individuals were considered in all analyses.

\section{Results}

The proportion of women was higher in all years of study and the largest population group was aged between 18 and 29 years, with a greater number of individuals aged 60 years or older in 2013. In all years, the majority of the population declared themselves as belonging to white color or race, but in 2013, blacks and browns together represented more than half of the population studied (Table 1). The relative frequency of individuals with low education has decreased over the years and there has been an increase in the proportion of people with university education. The possession of a health plan and registration with FHT increased over the study period, reaching approximately 30 and $60 \%$ of the population respectively, in 2013(Table 1).
Over the years, there has been an increase in the proportion of people who rated their health as good, reaching $56 \%$ in 2013, and the proportion of people who reported limitation in their usual activities was on an average $8 \%$ during the study period. Approximately 20\% of the population was classified as having multimorbidity during the study period. The demand for health services increased from 13\% in 1998 to 17\% in 2013. Medical consultations increased by 14\% between 1998 and 2013 . Finally, the percentage of hospitalisations decreased over the years, reaching 7\% in 2013 (Table 1).

Table 2 shows the prevalence of searching health services in the last 15 days according to the multimorbidity classification. Among individuals with multimorbidity, the prevalence of seeking services was higher among women, older age groups, whites, and browns, those with the lowest education levels, those who had health insurance and registration in primary care FHT. The prevalence of searching services was also higher among individuals who rated their health as very poor and presented limitations in their usual activities in the last 15 days. Also, an increase in the prevalence of searching for services over the years for all variables could be noticed, regardless of the multimorbidity classification. However, the prevalence of searching health services among individuals with multimorbidity was almost double that of individuals without multimorbidity, except for those who reported limitations on their usual activities (Table 2).

The prevalence of medical consultations in the last 12 months between 1998 and 2013, showed an increasing trend over the years for all variables analysed. The prevalence of medical appointments did not differ much with regard to sex, education, having health insurance, and limiting usual activities in the last 15 days among individuals who were classified as having multimorbidity. However, when comparing the prevalence of use for these same variables among individuals without multimorbidity, higher prevalence was observed among women, individuals with higher levels of education, individuals having a health plan, and individuals who reported limitation in the usual activities in the last 15 days (Table 3).

The prevalence of hospitalisations decreased over the study period, however, the prevalence of hospitalisation among those individuals characterised with multimorbidity was double that of the individuals without multimorbidity. The prevalence of hospitalisations among men with multimorbidity was higher compared to women in 2003 and almost triple compared to men without multimorbidity in 2013. The highest prevalence of hospitalisations among individuals with multimorbidity was at the lowest educational levels. Finally, the differences in the prevalence of hospitalisations according to possession of a health plan decreased over the years 
Table 1 Demographic, socioeconomic, multimorbidity, and health services characteristics of the study population by year. Brazil, 1998-2013

\begin{tabular}{|c|c|c|c|c|}
\hline \multirow[t]{3}{*}{ Variables } & 1998 & 2003 & 2008 & 2013 \\
\hline & $N=100,222,274$ & $N=118,463,739$ & $N=134,105,731$ & $N=124,010,200$ \\
\hline & $\%(95 \% \mathrm{Cl})$ & $\%(95 \% \mathrm{Cl})$ & $\%(95 \% \mathrm{Cl})$ & $\%(95 \% \mathrm{Cl})$ \\
\hline \multicolumn{5}{|l|}{ Sex } \\
\hline Male & $47.9(47.7-48.2)$ & $47.7(47.5-47.9)$ & $47.7(47.5-47.9)$ & $47.1(46.7-47.5)$ \\
\hline Female & $52.1(51.8-52.3)$ & $52.3(52.1-52.5)$ & $52.3(52.1-52.5)$ & $52.9(52.5-53.3)$ \\
\hline \multicolumn{5}{|l|}{ Age } \\
\hline $18-29$ & $32.5(32.3-32.7)$ & $32.3(32.1-32.5)$ & $29.7(29.5-29.9)$ & $26.5(26.1-26.9)$ \\
\hline $30-39$ & $23.4(23.2-23.6)$ & $22.2(22.0-22.3)$ & $21.2(21.0-21.3)$ & $21.1(20.8-21.5)$ \\
\hline $40-49$ & $18.3(18.1-18.5)$ & $18.6(18.4-18.7)$ & $19.1(19.0-19.3)$ & $18.6(18.2-18.9)$ \\
\hline $50-59$ & $12.0(11.8-12.1)$ & $12.6(12.4-12.7)$ & $14.1(14.0-14.3)$ & $15.7(15.4-16.0)$ \\
\hline $60-69$ & $8.0(7.8-8.1)$ & $8.0(7.9-8.1)$ & $8.8(8.7-8.9)$ & $10.1(10.0-10.4)$ \\
\hline$>=70$ & $5.9(5.8-6.0)$ & $6.4(6.3-6.5)$ & $7.1(7.0-7.2)$ & $8.0(7.7-8.2)$ \\
\hline \multicolumn{5}{|l|}{ Race/skin color } \\
\hline White & $56.4(56.1-56.6)$ & $53.9(53.7-54.1)$ & $50.0(49.8-50.2)$ & $47.6(47.2-47.9)$ \\
\hline Brown & $36.6(36.4-36.8)$ & $39.0(38.8-39.2)$ & $41.5(41.3-41.7)$ & $42.0(41.6-42.4)$ \\
\hline Black & $6.2(6.1-6.3)$ & $6.4(6.3-6.5)$ & $7.6(7.4-7.7)$ & $9.2(8.9-9.4)$ \\
\hline Yellow & $0.7(0.6-0.7)$ & $0.5(0.5-0.6)$ & $0.7(0.6-0.7)$ & $0.9(0.8-0.9)$ \\
\hline Indigenous & $0.2(0.2-0.2)$ & $0.2(0.2-0.2)$ & $0.3(0.3-0.3)$ & $0.4(0.4-0.5)$ \\
\hline \multicolumn{5}{|l|}{ Education } \\
\hline No schooling & $5.2(5.1-5.3)$ & $5.1(5.0-5.2)$ & $5.4(5.3-5.5)$ & $13.7(13.5-14.0)$ \\
\hline Incomplete elementary school & $52.2(52.0-52.5)$ & $44.3(44.1-44.5)$ & $36.2(36.0-36.5)$ & $25.6(25.3-26.0)$ \\
\hline Complete elementary school & $8.9(8.7-9.0)$ & $8.7(8.5-8.8)$ & $9.1(9.0-9.2)$ & $9.8(9.6-10.1)$ \\
\hline Incomplete high school & $7.8(7.7-7.9)$ & $8.5(8.4-8.6)$ & $8.1(8.0-8.2)$ & $5.4(5.3-5.6)$ \\
\hline Complete high school & $15.6(15.4-15.8)$ & $20.3(20.1-20.5)$ & $24.9(24.7-25.1)$ & $27.5(27.1-27.9)$ \\
\hline Incomplete university & $3.8(3.7-3.9)$ & $5.5(5.4-5.6)$ & $6.6(6.5-6.7)$ & $5.2(5.0-5.4)$ \\
\hline Completed university & $6.6(6.5-6.7)$ & $7.6(7.5-7.8)$ & $9.8(9.7-10.0)$ & $12.1(12.4-13.0)$ \\
\hline \multicolumn{5}{|l|}{ Health insurance } \\
\hline Yes & $26.6(26.4-26.8)$ & $26.9(26.7-27.1)$ & $28.1(27.9-28.3)$ & $29.9(29.5-30.2)$ \\
\hline No & $73.4(73.2-73.6)$ & $73.172 .9-73.3)$ & $71.9(71.7-72.1)$ & $70.1(69.8-70.5)$ \\
\hline \multicolumn{5}{|l|}{ Registered in FHT } \\
\hline Yes & - & - & $48.8(48.6-48.9)$ & $61.1(60.7-61.4)$ \\
\hline No & - & - & $51.3(51.1-51.4)$ & $38.9(38.6-39.3)$ \\
\hline \multicolumn{5}{|l|}{ Health self-assessment } \\
\hline Very good & $22.3(22.1-22.5)$ & $20.6(20.4-20.7)$ & $18.5(18.3-18.7)$ & $12.6(12.3-12.9)$ \\
\hline Good & $49.3(49.1-49.6)$ & $51.8(51.6-52.0)$ & $52.8(52.6-53.0)$ & $56.1(55.7-56.5)$ \\
\hline Regular & $23.1(22.9-23.3)$ & $22.9(22.7-23.1)$ & $23.6(23.4-23.7)$ & $25.7(25.4-26.1)$ \\
\hline Poor & $4.4(4.3-4.5)$ & $3.9(3.8-4.0)$ & $4.1(4.0-4.2)$ & $4.6(4.4-4.7)$ \\
\hline Very poor & $0.9(0.8-0.9)$ & $0.8(0.8-0.8)$ & $1.0(1.0-1.1)$ & $1.0(0.9-1.1)$ \\
\hline \multicolumn{5}{|l|}{ Limitation of usual activities } \\
\hline Yes & $7.2(7.1-7.3)$ & $7.3(7.2-7.4)$ & $8.9(8.8-9.0)$ & $7.5(7.3-7.8)$ \\
\hline No & $92.8(92.7-92.9)$ & $92.7(92.6-92.8)$ & $91.1(91.0-91.2)$ & $92.5(92.2-92.7)$ \\
\hline \multicolumn{5}{|l|}{ Multimorbidity } \\
\hline Yes & $21.5(21.3-21.7)$ & $18.1(18.0-18.3)$ & $18.5(18.3-18.6)$ & $22.1(21.6-22.7)$ \\
\hline
\end{tabular}


Table 1 Demographic, socioeconomic, multimorbidity, and health services characteristics of the study population by year. Brazil, 1998-2013 (Continued)

\begin{tabular}{|c|c|c|c|c|}
\hline \multirow[t]{3}{*}{ Variables } & 1998 & 2003 & 2008 & 2013 \\
\hline & $N=100,222,274$ & $N=118,463,739$ & $N=134,105,731$ & $N=124,010,200$ \\
\hline & $\%(95 \% \mathrm{Cl})$ & $\%(95 \% \mathrm{Cl})$ & $\%(95 \% \mathrm{Cl})$ & $\%(95 \% \mathrm{Cl})$ \\
\hline No & $78.5(78.3-78.7)$ & $81.9(81.7-82.0)$ & $81.5(81.4-81.7)$ & $77.9(77.3-78.4)$ \\
\hline \multicolumn{5}{|c|}{ Search for health services } \\
\hline Yes & $13.2(13.0-13.3)$ & $14.8(14.7-15.0)$ & $14.1(13.9-14.2)$ & $19.8(19.3-20.4)$ \\
\hline No & $86.8(86.7-87.0)$ & $85.2(85.0-85.3)$ & $85.9(85.8-86.1)$ & $80.2(79.6-80.7)$ \\
\hline \multicolumn{5}{|c|}{ Medical appointment } \\
\hline Yes & $57.6(57.3-57.8)$ & $64.6(64.4-64.8)$ & $69.8(69.7-70.0)$ & $78.6(78.0-79.1)$ \\
\hline No & $42.4(42.2-42.7)$ & $35.4(35.2-35.6)$ & $30.2(30.0-30.4)$ & $21.4(20.9-22.0)$ \\
\hline \multicolumn{5}{|c|}{ Hospitalisations } \\
\hline Yes & $8.4(8.3-8.6)$ & $8.1(7.9-8.2)$ & $8.0(7.9-8.1)$ & $7.9(7.6-8.3)$ \\
\hline No & $91.6(91.4-91.7)$ & $91.9(91.8-92.1)$ & $92.0(91.9-92.1)$ & $92.1(91.7-92.4)$ \\
\hline
\end{tabular}

among individuals who had multimorbidity, reaching less than $1 \%$ in the last year of study (Table 4 ).

The crudes and adjusted PRs for the use of health services (search for services, medical consultations, and hospitalisations) for each year of study stratified by sex are shown in Table 5. It is possible to verify that having multimorbidity was associated with a threefold increase in the probability of searching health care among men and a twofold increase for women in 1998.

Behind progressive adjustment due to predisposing factors, enabling factors and health needs, a reduction in the strength of association between the search for services and multimorbidity was observed over the years, regardless of gender. However, for men having multimorbidity, the search for services increased by $38 \%$ in 1998 and $46 \%$ in 2013. On the contrary, among women, having multimorbidity increased the search by $47 \%$ in 1998 and decreased to $39 \%$ in 2013.

Having multimorbidity increased the chance of having medical appointments in the past 12 months, but the association has decreased over the years, regardless of gender. After adjustment, for men, having multimorbidity increased the chance of medical consultations in 1998 by 28\%, reducing to $16 \%$ in 2013 . Among women, this association was 19\% in 1998 and $11 \%$ in 2013 (Table 5).

Regarding hospitalisations, in the bivariate analysis, the chance of being hospitalised among men who had multimorbidity was approximately 4 times greater compared to those who did not have multimorbidity in 1998. After progressive adjustment, there was a reduction in the association between having multimorbidity and having been hospitalised, regardless of gender. Among men, having multimorbidity increased the chance of having been hospitalised by $63 \%$ in 2003 , decreasing to $55 \%$ in 2013. In women, this association was $44 \%$ higher among those with multimorbidity in 2003, and 45\% in 2013 (Table 5).

\section{Discussion}

There was an increase in the prevalence of searching health services and medical appointments in the last 12 months between 1998 and 2013, regardless of the multimorbidity classification. On the other hand, there was a reduction in the prevalence of hospitalisations during the study period. Despite the similar trend of growth in the prevalence of searching health services, among individuals with multimorbidity, there is twice as high prevalence to those without multimorbidity for all the conditions studied, except for those who reported limitation in their usual activities in the last 15 days. However, for medical consultations, the prevalence rates did not differ with regard to sex, education, having a health plan and limiting usual activities in the last 15 days among individuals with multimorbidity.

Besides, it is noteworthy that despite the reduction in hospitalisations over the years, the prevalence of hospitalisations among men with multimorbidity was higher compared to women from 2003 and almost threefold compared to men without multimorbidity in 2013. Having multimorbidity increased the search for health services by $46 \%$ for men and $39 \%$ for women in the last year of study. This relationship increased the chances for medical appointments by $16 \%$ for men and $11 \%$ for women in 2013. Finally, having multimorbidity increased the chance of being hospitalised by $55 \%$ for men and 45\% for women in the year 2013.

In Brazil, NCD carriers use health services more [25]. Access to and use of health services depend on a set of factors that can be divided into determinants of supply and demand [26]. The perceived need, that is, the identification of a problem by the user, is the most important 
Table 2 Prevalence of searching health services in the last 15 days stratified by multimorbidity. Brazil, 1998-2013

\begin{tabular}{|c|c|c|c|c|c|c|c|c|c|c|}
\hline \multirow[t]{3}{*}{ Variables } & \multicolumn{5}{|c|}{ Multimorbidity } & \multicolumn{5}{|c|}{ Without multimorbidity } \\
\hline & \multirow{2}{*}{$\begin{array}{l}1998 \\
\%\end{array}$} & \multirow{2}{*}{$\begin{array}{l}2003 \\
\%\end{array}$} & \multirow{2}{*}{$\begin{array}{l}2008 \\
\%\end{array}$} & \multicolumn{2}{|l|}{2013} & \multirow{2}{*}{$\begin{array}{l}1998 \\
\%\end{array}$} & \multirow{2}{*}{$\begin{array}{l}2003 \\
\%\end{array}$} & \multirow{2}{*}{$\begin{array}{l}2008 \\
\%\end{array}$} & \multirow{2}{*}{$\begin{array}{l}2013 \\
\%\end{array}$} & \multirow[b]{2}{*}{$p$-value ${ }^{\mathrm{a}}$} \\
\hline & & & & $\%$ & $p$-value ${ }^{a}$ & & & & & \\
\hline & 25.95 & 31.54 & 29.95 & 34.44 & $* * *$ & 9.64 & 11.15 & 10.5 & 15.65 & $* * *$ \\
\hline \multicolumn{11}{|l|}{ Sex } \\
\hline Male & 20.57 & 27.17 & 26.8 & 29.88 & $* * *$ & 6.67 & 7.77 & 8.01 & 11.86 & $* * *$ \\
\hline Female & 29.05 & 33.89 & 31.71 & 36.48 & $* * *$ & 12.73 & 14.59 & 13.01 & 18.58 & $* * *$ \\
\hline \multicolumn{11}{|l|}{ Age } \\
\hline $18-29$ & 22.49 & 28.4 & 28.77 & 33.7 & $* * *$ & 8.82 & 9.68 & 9.27 & 13.25 & $* * *$ \\
\hline $30-39$ & 23.87 & 28.31 & 27.82 & 34.13 & $* * *$ & 9.34 & 10.9 & 10.04 & 14.06 & $* * *$ \\
\hline $40-49$ & 25.6 & 30.84 & 30 & 34.26 & $* * *$ & 9.94 & 11.91 & 10.76 & 15.58 & $* * *$ \\
\hline $50-59$ & 25.84 & 31.86 & 30.73 & 35.41 & $* * *$ & 10.69 & 12.4 & 11.96 & 16.31 & $* * *$ \\
\hline $60-69$ & 27.78 & 32.68 & 29.35 & 32.82 & $* * *$ & 11.81 & 14.18 & 12.54 & 20.77 & $* * *$ \\
\hline$>=70$ & 28.07 & 33.9 & 30.89 & 35.57 & $* * *$ & 13.93 & 16.14 & 15.33 & 22.23 & $* * *$ \\
\hline \multicolumn{11}{|l|}{ Race/skin color } \\
\hline White & 27.44 & 32.35 & 29.93 & 35.54 & $* * *$ & 10.07 & 11.59 & 10.93 & 16.01 & $* * *$ \\
\hline Brown & 25.26 & 31.88 & 30.77 & 36.33 & $* * *$ & 9.15 & 11.35 & 10.43 & 14.4 & $* * *$ \\
\hline Black & 25.58 & 26.84 & 22.56 & 41.03 & & 7.63 & 10.51 & 11.04 & 19.03 & $* *$ \\
\hline Yellow & 23.84 & 30.28 & 30.05 & 31.9 & $* * *$ & 9.08 & 10.53 & 9.98 & 15.44 & $* * *$ \\
\hline Indigenous & 35.37 & 28.09 & 25.95 & 56.17 & & 10.85 & 12.68 & 13.93 & 12.06 & \\
\hline \multicolumn{11}{|l|}{ Education } \\
\hline No schooling & 24.21 & 33.36 & 31.38 & 33.95 & $* * *$ & 8.55 & 11.27 & 10.89 & 16.34 & $* * *$ \\
\hline Incomplete elementary school & 26.39 & 31.54 & 30.19 & 33.42 & $* * *$ & 9.02 & 10.91 & 10.51 & 16.59 & $* * *$ \\
\hline Complete elementary school & 28.02 & 30.63 & 30.42 & 40.36 & $* * *$ & 9.44 & 10.2 & 10.01 & 13.43 & $* * *$ \\
\hline Incomplete high school & 26.33 & 29.85 & 29.3 & 27.52 & & 9.48 & 9.65 & 9.42 & 13.96 & $* *$ \\
\hline Complete high school & 27.72 & 30.53 & 29.53 & 34.7 & $* *$ & 10.56 & 11.37 & 10.09 & 14.6 & $* * *$ \\
\hline Incomplete university & 24.45 & 29.27 & 27.92 & 35.07 & & 11.33 & 12.02 & 10 & 15.07 & $* * *$ \\
\hline Completed university & 31.04 & 32.38 & 28.67 & 34.65 & $* *$ & 12.72 & 13.9 & 12.39 & 17.93 & $* * *$ \\
\hline \multicolumn{11}{|l|}{ Health insurance } \\
\hline Yes & 32.4 & 35.14 & 32.32 & 36.89 & $* * *$ & 14.0 & 15.1 & 13.3 & 18.85 & $* * *$ \\
\hline No & 23.89 & 30.03 & 28.93 & 33.18 & $* * *$ & 8.0 & 9.74 & 9.42 & 14.11 & $* * *$ \\
\hline \multicolumn{11}{|l|}{ Registered in FHT } \\
\hline Yes & - & - & 30.96 & 34.12 & & - & - & 10.87 & 16.23 & \\
\hline No & - & - & 28.91 & 34.16 & & - & - & 10.15 & 15.49 & \\
\hline \multicolumn{11}{|l|}{ Health self-assessment } \\
\hline Very good & 14.92 & 19.32 & 18.25 & 23.79 & $* * *$ & 6.24 & 7.33 & 6.93 & 12.66 & $* * *$ \\
\hline Good & 16.34 & 21.83 & 21.16 & 27.19 & $* * *$ & 8.4 & 9.52 & 8.78 & 13.25 & $* * *$ \\
\hline Regular & 26.79 & 32.82 & 30.66 & 36.31 & $* * *$ & 18.0 & 19.65 & 18.33 & 22.35 & $* * *$ \\
\hline Poor & 39.1 & 46.95 & 41.64 & 45.05 & $* * *$ & 26.54 & 29.56 & 28.51 & 26.14 & * \\
\hline Very poor & 47.7 & 56.48 & 51.1 & 49.27 & $* * *$ & 29.27 & 29.43 & 34.15 & 35.99 & \\
\hline \multicolumn{11}{|l|}{ Limitation of usual activities } \\
\hline Yes & 58.05 & 65 & 60.24 & 62.37 & $* * *$ & 56.08 & 56.87 & 52.23 & 63.36 & $* * *$ \\
\hline No & 18.8 & 23.63 & 21.37 & 27.8 & $* * *$ & 7.62 & 8.89 & 7.86 & 12.39 & $* * *$ \\
\hline
\end{tabular}

Note: ${ }^{a}$ : Test Wald (Pearson) adjusted for the linear tendency; $p$-value: ${ }^{*} \leq 0.05,{ }^{* *} \leq 0.01,{ }^{* *} \leq 0.001$ 
Table 3 Prevalence of medical appointments in the last 12 months stratified by multimorbidity. Brazil, 1998-2013

\begin{tabular}{|c|c|c|c|c|c|c|c|c|c|c|}
\hline \multirow[t]{3}{*}{ Variables } & \multicolumn{5}{|c|}{ Multimorbidity } & \multicolumn{5}{|c|}{ Without multimorbidity } \\
\hline & \multirow{2}{*}{$\begin{array}{l}1998 \\
\%\end{array}$} & \multirow{2}{*}{$\begin{array}{l}2003 \\
\%\end{array}$} & \multirow{2}{*}{$\begin{array}{l}2008 \\
\%\end{array}$} & \multicolumn{2}{|l|}{2013} & \multirow{2}{*}{$\begin{array}{l}1998 \\
\%\end{array}$} & \multirow{2}{*}{$\begin{array}{l}2003 \\
\%\end{array}$} & \multirow{2}{*}{$\begin{array}{l}2008 \\
\%\end{array}$} & \multirow{2}{*}{$\begin{array}{l}2013 \\
\%\end{array}$} & \multirow[b]{2}{*}{ p-value } \\
\hline & & & & $\%$ & $p$-value ${ }^{a}$ & & & & & \\
\hline & 79.47 & 87.62 & 89.94 & 92.36 & $* * *$ & 51.56 & 59.47 & 65.29 & 74.64 & $* * *$ \\
\hline Mean annual of consultations & 5.8 & 6.6 & 6.7 & 6.0 & & 3.5 & 3.6 & 3.7 & 3.6 & \\
\hline \multicolumn{11}{|l|}{ Sex } \\
\hline Male & 71.38 & 82.19 & 85.48 & 88.23 & $* * *$ & 40.82 & 47.83 & 53.66 & 67.2 & $* * *$ \\
\hline Female & 84.14 & 90.53 & 92.44 & 94.21 & $* * *$ & 62.75 & 71.38 & 77.08 & 80.39 & $* * *$ \\
\hline \multicolumn{11}{|l|}{ Age } \\
\hline $18-29$ & 75.76 & 83.8 & 86.54 & 84.52 & $* * *$ & 47.65 & 55.11 & 60.99 & 73.04 & $* * *$ \\
\hline $30-39$ & 77.01 & 84.98 & 87.7 & 90.47 & $* * *$ & 52.13 & 60.08 & 65.99 & 73.56 & $* * *$ \\
\hline $40-49$ & 77.86 & 86.51 & 88.9 & 90.69 & $* * *$ & 53.33 & 62.05 & 67.19 & 73.13 & $* * *$ \\
\hline $50-59$ & 79.63 & 87.61 & 89.96 & 92.13 & $* * *$ & 55.39 & 62.95 & 68.24 & 75.25 & $* * *$ \\
\hline $60-69$ & 81.97 & 89.3 & 90.82 & 94.01 & $* * *$ & 59.24 & 65.1 & 69.94 & 79.1 & $* * *$ \\
\hline$>=70$ & 82.54 & 90.1 & 91.83 & 94.63 & $* * *$ & 61.94 & 69.63 & 74.29 & 81.68 & $* * *$ \\
\hline \multicolumn{11}{|l|}{ Race/skin color } \\
\hline White & 81.78 & 88.47 & 90.85 & 93.39 & $* * *$ & 54.1 & 61.96 & 67.46 & 76.45 & $* * *$ \\
\hline Brown & 79.57 & 87.79 & 89.96 & 91.27 & $* * *$ & 47 & 57.68 & 64.58 & 72.65 & $* * *$ \\
\hline Black & 84.75 & 87.49 & 88.18 & 91.17 & & 50.91 & 61.47 & 65.88 & 73.56 & $* * *$ \\
\hline Yellow & 75.99 & 86.24 & 88.74 & 91.01 & $* * *$ & 48.37 & 56.39 & 62.93 & 72.9 & $* * *$ \\
\hline Indigenous & 79.38 & 84.57 & 87.7 & 96.51 & $* *$ & 50.17 & 61.65 & 64.36 & 81.01 & $* * *$ \\
\hline \multicolumn{11}{|l|}{ Education } \\
\hline No schooling & 73.59 & 86.01 & 88.22 & 92.07 & $* * *$ & 45.06 & 51.27 & 57.53 & 70.57 & $* * *$ \\
\hline Incomplete elementary school & 80.35 & 87.55 & 90.2 & 92.03 & $* * *$ & 48.36 & 56.5 & 62.32 & 71.82 & $* * *$ \\
\hline Complete elementary school & 82.95 & 88.3 & 89.35 & 94.32 & $* * *$ & 50.48 & 57.91 & 63.4 & 72.27 & $* * *$ \\
\hline Incomplete high school & 80.38 & 87.61 & 91.02 & 89.97 & $* * *$ & 50.92 & 56.61 & 61.04 & 72.04 & $* * *$ \\
\hline Complete high school & 83.44 & 88.08 & 90.83 & 91.02 & $* * *$ & 57.98 & 63.07 & 67.34 & 75.06 & $* * *$ \\
\hline Incomplete university & 84.09 & 88.85 & 89.17 & 92.17 & * & 61.45 & 67.99 & 70.04 & 76.28 & $* * *$ \\
\hline Completed university & 88.84 & 91.96 & 93.14 & 94.86 & $* * *$ & 67.63 & 75.17 & 78.5 & 83.1 & $* * *$ \\
\hline \multicolumn{11}{|l|}{ Health insurance } \\
\hline Yes & 89.27 & 93.07 & 94.31 & 96.59 & $* * *$ & 69 & 75.79 & 78.45 & 86.44 & $* * *$ \\
\hline No & 76.35 & 85.32 & 88.07 & 90.18 & $* * *$ & 45.01 & 53.66 & 60.24 & 68.97 & $* * *$ \\
\hline \multicolumn{11}{|l|}{ Registered in FHT } \\
\hline Yes & - & - & 90.04 & 92.15 & & - & - & 64.56 & 75.19 & \\
\hline No & - & - & 89.84 & 92.74 & & - & - & 66 & 74.81 & \\
\hline \multicolumn{11}{|l|}{ Health self-assessment } \\
\hline Very good & 68.25 & 81.56 & 85 & 89.48 & $* * *$ & 43.35 & 53.36 & 59.17 & 71.97 & $* * *$ \\
\hline Good & 71.92 & 83.24 & 87.43 & 89.61 & $* * *$ & 50.4 & 57.53 & 63.79 & 72.86 & $* * *$ \\
\hline Regular & 81.42 & 89.04 & 90.44 & 93.48 & $* * *$ & 67.6 & 72.28 & 75.9 & 79.97 & $* * *$ \\
\hline Poor & 87.38 & 92.52 & 93.08 & 94.68 & $* * *$ & 74.84 & 78.19 & 82.05 & 83.24 & $* * *$ \\
\hline Very poor & 89.83 & 94.67 & 94.3 & 97.18 & $* * *$ & 70.38 & 77.39 & 82.36 & 82.56 & $* * *$ \\
\hline \multicolumn{11}{|l|}{ Limitation of usual activities } \\
\hline Yes & 90.93 & 95.39 & 95.51 & 95.46 & $* * *$ & 84 & 86.55 & 86.77 & 93.47 & $* * *$ \\
\hline No & 76.92 & 85.78 & 88.36 & 91.62 & $* * *$ & 50.15 & 58.13 & 63.93 & 73.35 & $* * *$ \\
\hline
\end{tabular}

Note: ${ }^{a}$ : Test Wald (Pearson) adjusted for the linear tendency; $p$-value: ${ }^{*} \leq 0.05,{ }^{* *} \leq 0.01,{ }^{* * *} \leq 0.001$ 
Table 4 Prevalence of hospitalisations in the last 12 months stratified by multimorbidity. Brazil, 1998-2013

\begin{tabular}{|c|c|c|c|c|c|c|c|c|c|c|}
\hline \multirow[t]{3}{*}{ Variables } & \multicolumn{5}{|c|}{ Multimorbidity } & \multicolumn{5}{|c|}{ Without multimorbidity } \\
\hline & \multirow{2}{*}{$\begin{array}{l}1998 \\
\%\end{array}$} & \multirow{2}{*}{$\begin{array}{l}2003 \\
\%\end{array}$} & \multirow{2}{*}{$\begin{array}{l}2008 \\
\%\end{array}$} & \multicolumn{2}{|l|}{2013} & \multirow{2}{*}{$\begin{array}{l}1998 \\
\%\end{array}$} & \multirow{2}{*}{$\begin{array}{l}2003 \\
\%\end{array}$} & \multirow{2}{*}{$\begin{array}{l}2008 \\
\%\end{array}$} & \multirow{2}{*}{$\begin{array}{l}2013 \\
\%\end{array}$} & \multirow[b]{2}{*}{ p-value ${ }^{a}$} \\
\hline & & & & $\%$ & p-value ${ }^{a}$ & & & & & \\
\hline & 15.03 & 15.74 & 15.68 & 13.44 & $* * *$ & 6.62 & 6.34 & 6.29 & 6.38 & $* * *$ \\
\hline Mean annual of hospitalisations & 1.6 & 1.7 & 1.7 & 1.7 & & 1.2 & 1.3 & 1.3 & 1.3 & \\
\hline \multicolumn{11}{|l|}{ Sex } \\
\hline Male & 14.44 & 16.21 & 16.21 & 13.87 & $* * *$ & 3.95 & 4.24 & 4.50 & 4.78 & $* * *$ \\
\hline Female & 15.37 & 15.49 & 15.39 & 13.24 & $* *$ & 9.41 & 8.50 & 8.11 & 7.63 & $* * *$ \\
\hline \multicolumn{11}{|l|}{ Age } \\
\hline $18-29$ & 17.41 & 17.57 & 17.78 & 15.8 & & 7.57 & 6.79 & 6.93 & 7.05 & $* * *$ \\
\hline $30-39$ & 13.02 & 14.02 & 14.82 & 11.7 & * & 6.27 & 6.41 & 6.24 & 6.44 & \\
\hline $40-49$ & 12.18 & 13.6 & 14.25 & 11.1 & $* * *$ & 4.78 & 5.19 & 5.07 & 5.23 & \\
\hline $50-59$ & 13.62 & 14.38 & 14.27 & 11.14 & $* *$ & 5.37 & 5.19 & 5.30 & 5.92 & \\
\hline $60-69$ & 15.67 & 15.43 & 14.81 & 14.36 & & 7.53 & 6.34 & 5.96 & 6.07 & $* * *$ \\
\hline$>=70$ & 20.46 & 20.42 & 19.54 & 17.17 & & 10.29 & 10.20 & 10.19 & 8.67 & \\
\hline \multicolumn{11}{|l|}{ Race/skin color } \\
\hline White & 15.34 & 15.67 & 15.66 & 13.00 & $* *$ & 6.63 & 6.20 & 6.23 & 6.27 & $* * *$ \\
\hline Brown & 13.62 & 15.68 & 15.35 & 13.78 & & 5.86 & 6.13 & 5.88 & 5.65 & \\
\hline Black & 17.74 & 12.15 & 11.5 & 13.18 & & 4.44 & 6.02 & 6.06 & 7.84 & \\
\hline Yellow & 14.8 & 15.9 & 15.86 & 14.02 & $* *$ & 6.76 & 6.57 & 6.44 & 6.67 & \\
\hline Indigenous & 16.46 & 17.27 & 20.38 & 14.29 & & 7.97 & 7.53 & 8.16 & 4.60 & \\
\hline \multicolumn{11}{|l|}{ Education } \\
\hline No schooling & 15.68 & 18.14 & 18.7 & 17.36 & $* *$ & 7.66 & 7.69 & 6.88 & 8.31 & \\
\hline Incomplete elementary school & 15.08 & 15.35 & 15.08 & 13.04 & & 6.96 & 6.50 & 6.62 & 5.95 & $* *$ \\
\hline Complete elementary school & 13.33 & 12.95 & 15.6 & 12.59 & * & 6.55 & 6.02 & 6.58 & 6.62 & \\
\hline Incomplete high school & 13.46 & 15.00 & 14.66 & 11.91 & & 5.23 & 5.89 & 6.17 & 7.96 & $* * *$ \\
\hline Complete high school & 12.92 & 14.14 & 13.86 & 11.06 & & 6.43 & 6.13 & 5.82 & 6.02 & * \\
\hline Incomplete university & 13.85 & 13.22 & 15.35 & 11.34 & & 5.04 & 4.81 & 5.04 & 5.29 & \\
\hline Completed university & 13.88 & 15.15 & 14.76 & 12.81 & & 5.87 & 6.14 & 6.36 & 5.95 & \\
\hline \multicolumn{11}{|l|}{ Health insurance } \\
\hline Yes & 16.96 & 17.18 & 17.05 & 12.84 & $* * *$ & 7.56 & 7.20 & 7.08 & 7.38 & \\
\hline No & 14.42 & 15.13 & 15.1 & 13.74 & $* *$ & 6.27 & 6.04 & 5.99 & 5.90 & * \\
\hline \multicolumn{11}{|l|}{ Registered in FHT } \\
\hline Yes & - & - & 16.68 & 14.17 & & - & - & 6.86 & 6.76 & \\
\hline No & - & - & 14.65 & 12.43 & & - & - & 5.75 & 5.87 & \\
\hline \multicolumn{11}{|l|}{ Health self-assessment } \\
\hline Very good & 7.66 & 8.91 & 9.35 & 11.00 & & 4.71 & 4.50 & 4.34 & 5.10 & $*$ \\
\hline Good & 8.84 & 9.61 & 9.99 & 9.99 & * & 5.89 & 5.57 & 5.47 & 5.60 & $* *$ \\
\hline Regular & 14.58 & 15.52 & 15.10 & 12.57 & $* * *$ & 10.85 & 9.85 & 9.81 & 8.33 & $* * *$ \\
\hline Poor & 26.20 & 27.54 & 25.33 & 20.52 & $* * *$ & 20.67 & 19.00 & 18.97 & 12.52 & $* * *$ \\
\hline Very poor & 33.37 & 37.98 & 34.44 & 32.52 & & 25.32 & 22.03 & 23.21 & 17.53 & \\
\hline \multicolumn{11}{|l|}{ Limitation of usual activities } \\
\hline Yes & 29.12 & 31.47 & 28.64 & 25.52 & $* * *$ & 22.94 & 21.91 & 20.08 & 19.48 & $* * *$ \\
\hline No & 11.89 & 12.02 & 12.01 & 10.56 & & 5.913 & 5.574 & 5.42 & 5.49 & $* * *$ \\
\hline
\end{tabular}

Note: ${ }^{a}$ : Test Wald (Pearson) adjusted for the linear tendency; $p$-value: ${ }^{*} \leq 0.05,{ }^{* *} \leq 0.01,{ }^{* * *} \leq 0.001$ 
Table 5 Crude and adjusted prevalence ratios for the use of health services. Brazil, 1998-2013

\begin{tabular}{|c|c|c|c|c|c|c|c|c|}
\hline \multirow[t]{3}{*}{ Variable } & \multicolumn{4}{|l|}{ Male } & \multicolumn{4}{|l|}{ Female } \\
\hline & Model 1 & Model 2 & Model 3 & Model 4 & Model 1 & Model 2 & Model 3 & Model 4 \\
\hline & PR $(95 \% \mathrm{Cl})$ & PR $(95 \% \mathrm{Cl})$ & PR (95\% Cl) & PR $(95 \% \mathrm{Cl})$ & PR $(95 \% \mathrm{Cl})$ & PR $(95 \% \mathrm{Cl})$ & PR (95\% CI) & PR $(95 \% \mathrm{Cl})$ \\
\hline \multicolumn{9}{|c|}{ Search for health services } \\
\hline \multirow[t]{2}{*}{1998} & 3.08 & 2.66 & 2.57 & 1.38 & 2.28 & 2.21 & 2.24 & 1.47 \\
\hline & $(2.95-3.22)$ & $(2.52-2.81)$ & $(2.44-2.69)$ & $(1.31-1.45)$ & $(2.22-2.35)$ & $(2.14-2.29)$ & $(2.17-2.31)$ & $(1.42-1.52)$ \\
\hline \multirow[t]{2}{*}{2003} & 3.49 & 2.75 & 2.73 & 1.58 & 2.32 & 2.16 & 2.18 & 1.47 \\
\hline & $(3.37-3.62)$ & $(2.63-2.89)$ & $(2.61-2.85)$ & $(1.51-1.65)$ & $(2.27-2.38)$ & $(2.09-2.22)$ & $(2.12-2.24)$ & $(1.42-1.51)$ \\
\hline \multirow[t]{2}{*}{2008} & 3.34 & 2.67 & 2.66 & 1.51 & 2.44 & 2.35 & 2.34 & 1.52 \\
\hline & $(3.23-3.46)$ & $(2.55-2.80)$ & $(2.56-2.78)$ & $(1.45-1.58)$ & $(2.38-2.50)$ & $(2.28-2.43)$ & $(2.27-2.41)$ & $(1.48-1.57)$ \\
\hline \multirow[t]{2}{*}{2013} & 2.52 & 2.09 & 1.98 & 1.46 & 1.96 & 1.85 & 1.81 & 1.39 \\
\hline & $(2.26-2.81)$ & $(1.85-2.36)$ & $(1.75-2.25)$ & $(1.28-1.67)$ & $(1.84-2.10)$ & $(1.72-1.99)$ & $(1.68-1.96)$ & $(1.29-1.51)$ \\
\hline \multicolumn{9}{|c|}{ Doctor's appointments } \\
\hline \multirow[t]{2}{*}{1998} & 1.75 & 1.59 & 1.59 & 1.28 & 1.34 & 1.32 & 1.33 & 1.19 \\
\hline & $(1.72-1.77)$ & $(1.56-1.61)$ & $(1.56-1.61)$ & $(1.26-1.30)$ & $(1.33-1.35)$ & $(1.31-1.34)$ & $(1.31-1.34)$ & $(1.18-1.20)$ \\
\hline \multirow[t]{2}{*}{2003} & 1.72 & 1.52 & 1.50 & 1.28 & 1.27 & 1.23 & 1.22 & 1.14 \\
\hline & $(1.70-1.74)$ & $(1.50-1.54)$ & $(1.48-1.52)$ & $(1.26-1.30)$ & $(1.26-1.28)$ & $(1.22-1.24)$ & $(1.22-1.23)$ & $(1.14-1.15)$ \\
\hline \multirow[t]{2}{*}{2008} & 1.59 & 1.45 & 1.44 & 1.24 & 1.20 & 1.17 & 1.17 & 1.11 \\
\hline & $(1.58-1.61)$ & $(1.43-1.47)$ & $(1.42-1.45)$ & $(1.22-1.25)$ & $(1.19-1.21)$ & $(1.16-1.18)$ & $(1.16-1.17)$ & $(1.10-1.11)$ \\
\hline \multirow[t]{2}{*}{2013} & 1.31 & 1.25 & 1.23 & 1.16 & 1.17 & 1.16 & 1.15 & 1.11 \\
\hline & $(1.28-1.35)$ & $(1.21-1.29)$ & $(1.19-1.26)$ & $(1.12-1.20)$ & $(1.16-1.19)$ & $(1.14-1.18)$ & $(1.13-1.17)$ & $(1.09-1.12)$ \\
\hline \multicolumn{9}{|c|}{ Hospitalisations } \\
\hline \multirow[t]{2}{*}{1998} & 3.66 & 2.67 & 2.66 & 1.43 & 1.63 & 1.79 & 1.80 & 1.30 \\
\hline & $(3.47-3.86)$ & $(2.48-2.86)$ & $(2.48-2.86)$ & $(1.32-1.55)$ & $(1.57-1.70)$ & $(1.71-1.88)$ & $(1.72-1.88)$ & $(1.23-1.36)$ \\
\hline \multirow[t]{2}{*}{2003} & 3.83 & 2.79 & 2.76 & 1.63 & 1.82 & 2.05 & 2.02 & 1.44 \\
\hline & $(3.64-4.02)$ & $(2.61-2.98)$ & $(2.59-2.95)$ & $(1.52-1.75)$ & $(1.76-1.89)$ & $(1.96-2.14)$ & $(1.93-2.11)$ & $(1.37-1.51)$ \\
\hline \multirow[t]{2}{*}{2008} & 3.60 & 2.76 & 2.72 & 1.54 & 1.90 & 2.16 & 2.13 & 1.52 \\
\hline & $(3.43-3.78)$ & $(2.59-2.94)$ & $(2.55-2.90)$ & $(1.44-1.64)$ & $(1.83-1.97)$ & $(2.06-2.25)$ & $(2.04-2.23)$ & $(1.45-1.60)$ \\
\hline \multirow[t]{2}{*}{2013} & 2.90 & 2.19 & 2.18 & 1.55 & 1.74 & 1.85 & 1.83 & 1.45 \\
\hline & $(2.45-3.44)$ & $(1.83-2.63)$ & $(1.80-2.65)$ & $(1.26-1.90)$ & $(1.54-1.95)$ & $(1.64-2.10)$ & $(1.60-2.09)$ & $(1.25-1.68)$ \\
\hline
\end{tabular}

Note: Model 1: crude analysis; Model 2: adjusted for age, education and race/color; Model 3: Model $2+$ possession of a health insurance and FHT registration; Model 4: Model $3+$ self-assessment of health status and limitation of usual activities

driver of demand and usually overlaps other demographic and social characteristics [26, 27]. In the case of multimorbidity, our study showed that the prevalence of searching for services in the last 15 days was twice as high as those without multimorbidity, regardless of the sociodemographic characteristic analysed.

Also, the high prevalence of searching health services in this population can be explained in part, by the components of the provision of health services. In Brazil and most parts of the world, health systems are designed around unique conditions or body systems [28]. This focus extends to the training of health professionals, particularly those who work in hospitals where specialization is common, leaving the coordination of care for patients with multiple chronic conditions to family doctors, general practitioners and geriatricians [7, $28]$. This health care model can motivate a greater number of visits to different services by the same individual, overloading the health system.

Regarding medical consultations in the last 12 months, according to Viacava and Bellido (2016) [29] in 2013, $71.2 \%$ of the Brazilian population reported having had a medical consultation in the last 12 months; and in all regions of the country, except for the North, the increase in the prevalence of medical consultations was significant, between 1998 and 2013. In the general population, the use of health services is higher among adults with private insurance, among women and for people with a higher level of education in all years [30]. However, similar to our study, a study carried out in Serbia [31] found 
that having multimorbidity reduced the differences in the prevalence of medical visits in these variables, indicating a possible reduction in inequalities in the use of health services in populations with greater health needs, such as the case of people with multimorbidity.

The use of secondary services, measured as utilizing hospitalisations, had a prevalence twice as high among individuals classified as having multimorbidity and had a different pattern with regard to sex. In general, our results are in line with the findings of other studies, which point to a twice as high probability of hospitalisations among individuals with multimorbidity [13, 31-33].

Among the three health service utilization outcomes measured by this study, only the prevalence of hospitalisations was higher among men than women. According to Hulka and Wheat (1985) [34], the use of health services can be explained mainly by the profile of the health needs of a population group. It is already known that in the general population, women make more visits to primary care centres than men and seek more services for routine exams and prevention, while men seek health services predominantly due to illness [35, 36].

The study by Jankovic et al. (2018) [31], found a three times greater probability of having a medical consultation ( $\mathrm{OR}=3.17$ in men, $\mathrm{OR}=3.14$ in women) and two times greater probability of having been hospitalised in the last 12 months $(\mathrm{OR}=2.45$ in men, $\mathrm{OR}=1.97$ in women) in the Serbian population, among individuals with multimorbidity compared to those without any condition.

In our study, even after progressive adjustment of predisposing factors, enabling factors and health needs, having multimorbidity increased the chance of using health services for the three outcomes analysed, with greater influence among men. Our findings corroborate the results of other studies [12, 13, 15, 32, 37, 38], showing an increase in the use of health services in primary and secondary care associated with multimorbidity, even when controlling for age, sex and social status.

The study's limitations include the use of self-reported clinical conditions for chronic diseases and the use of health services that may underestimate their prevalence $[8,39]$. Furthermore, in defining multimorbidity as a simple count of NCDs, our study considered all diseases equally, although the effect of multimorbidity on individuals may vary with the combination and severity of NCDs [7]. Additionally, it should be noted that the list of self-reported morbidities used for the classification of simple count addressed only 10 diagnoses, a fact that may have reduced the estimates of multimorbidity among the individuals evaluated.

This study represents one of the first detailed descriptions of the effect of multimorbidity on the use of health services in Brazil. Among the strengths, this study included data of national scope that make it possible to generalise our results to the entire population and even to other countries with similar characteristics. Also, the analyses including four points in time made it possible to infer trends in the use of services, and the very similar issues in the 15 years analysed allowed to maintain comparability.

\section{Conclusion}

Multimorbidity is increasingly becoming common worldwide, with increasing implications for the management of patients, assessment of disease burden in populations and the efficiency and effectiveness of health systems [7, 40]. This study found that individuals with multimorbidity have higher levels of use of health services and, in the process, can often be seen by several health professionals.

To increase the quality of care provided to these patients and reduce the rising costs of health care, it is necessary to focus on continuous, coordinated and comprehensive approaches to the care of people with multimorbidity through the health system. This requires a change from current approaches, which often emphasize specific vertical disease programs.

Primary care services are the ideal setting for this process to occur, given their fundamental role in providing continuous, well-coordinated and comprehensive care to patients with complex health needs, including those with multiple NCDs.

Finally, more research is needed to better understand the epidemiology of multimorbidity and the associated impacts on the use and costs of health services in Brazil. Evidence on the patterns of use of health services contributes to the improvement of the health system, in the improvement of the management of individuals with multimorbidity aiming at better health results, thus increasing the efficiency of the assistance provided and reducing costs.

\section{Supplementary Information}

The online version contains supplementary material available at https://doi. org/10.1186/s12913-020-05938-4.

\section{Additional file 1.}

\section{Abbreviations}

NCDs: Chronic non-communicable diseases; PNAD: Brazilian National Household Sample Survey; PNS: Brazilian National Health Survey; IBGE: Brazilian Institute Geography and Statistics; FHT: Family health teams; PR: Prevalence ratios; $95 \% \mathrm{Cl}$ : 95\% confidence intervals; OR: Odds ratio 


\section{Authors' contributions}

ASSS and JUB contributed to the proposal development and write-up of the research, in data interpretation, critical review of the manuscript and approved the final version of the manuscript.

\section{Funding}

This study was financed in part by the Coordenação de Aperfeiçoamento de Pessoal de Nível Superior - Brasil (CAPES) and Research Support Foundation of the State of Rio de Janeiro (FAPERJ).

\section{Availability of data and materials}

The PNAD and PNS microdata are accessible to the public and available on the IBGE website.

- https://www.ibge.gov.br/estatisticas/sociais/saude/19898-suplementospnad $3 . h t m l ?=\& t=$ microdados

- https://www.ibge.gov.br/estatisticas/sociais/saude/9160-pesquisa-nacionalde-saude.html?=\&t=microdados

\section{Ethics approval and consent to participate}

The PNAD and PNS project was submitted and approved by the National Research Ethics Commission (CONEP). Survey microdata is available in open access and does not require administrative permission for use.

\section{Consent for publication}

Not applicable.

\section{Competing interests}

The authors declare that they have no competing interests.

\section{Author details}

'Programa de Pós-graduação em Saúde Coletiva, Instituto de Medicina Social, Universidade do Estado do Rio de Janeiro, Rio de Janeiro, RJ, Brazil. 2Departamento de Epidemiologia, Fundação Oswaldo Cruz, Escola Nacional de Saúde Pública Sergio Arouca, Rio de Janeiro, RJ, Brazil. ${ }^{3}$ Departamento de Epidemiologia, Instituto de Medicina Social, Universidade do Estado do Rio de Janeiro, Rio de Janeiro, RJ, Brazil.

Received: 26 August 2020 Accepted: 18 November 2020 Published online: 25 November 2020

\section{References}

1. Beaglehole R, Bonita R, Horton R, Adams C, Alleyne G, Asaria P, et al. Priority actions for the non-communicable disease crisis. Lancet. 2011;377(9775). https://doi.org/10.1016/S0140-6736(11)60393-0.

2. World Health Organization. Global action plan for the prevention and control of noncommunicable diseases 2013-2020: World Health Organization; 2013.

3. World Health Organization Global status report on noncommunicable diseases 2014. World Health Organization, 2014.

4. Johnston MC, Crilly M, Black C, Prescott GJ, Mercer SW. Defining and Measuring Multimorbidity: A Systematic Review of Systematic Reviews. Eur J Public Health. 2019;29(1). https://doi.org/10.1093/eurpub/cky098.

5. Nguyen H, Manolova G, Daskalopoulou C, Vitoratou S, Prince M, Prina AM. Prevalence of multimorbidity in community settings: A systematic review and meta-analysis of observational studies. J Comorb. 2019;9: 2235042x19870934. Epub 2019/09/07. https://doi.org/10.1177/ $2235042 \times 19870934$.

6. Rzewuska M, de Azevedo-Marques JM, Coxon D, Zanetti ML, Zanetti AC, Franco $\sqcup$, et al. Epidemiology of multimorbidity within the Brazilian adult general population: evidence from the 2013 National Health Survey (PNS 2013). PLoS One. 2017;12(2):e0171813. Epub 2017/02/10. https://doi.org/10. 1371/journal.pone.0171813.

7. Barnett K, Mercer SW, Norbury M, Watt G, Wyke S, Guthrie B. Epidemiology of multimorbidity and implications for health care, research, and medical education: a cross-sectional study. Lancet. 2012;380(9836):37-43. https://doi. org/10.1016/S0140-6736(12)60240-2

8. Fortin M, Stewart M, Poitras ME, Almirall J, Maddocks H. A systematic review of prevalence studies on multimorbidity: toward a more uniform methodology. Ann Fam Med. 2012;10(2):142-51. Epub 2012/03/14. https:// doi.org/10.1370/afm.1337.
9. DuGoff EH, Canudas-Romo V, Buttorff C, Leff B, Anderson GF. Multiple chronic conditions and life expectancy: a life table analysis. Med Care. 2014; 52(8):688-94. Epub 2014/07/16. https://doi.org/10.1097/mlr. 0000000000000166.

10. Fortin M, Lapointe L, Hudon C, Vanasse A, Ntetu AL, Maltais D. Multimorbidity and quality of life in primary care: a systematic review. Health Qual Life Outcomes. 2004;2:51. https://doi.org/10.1186/1477-7525-251.

11. Pati S, Agrawal S, Swain S, Lee JT, Vellakkal S, Hussain MA, et al. Non communicable disease multimorbidity and associated health care utilization and expenditures in India: cross-sectional study. BMC Health Serv Res. 2014; 14:451. Epub 2014/10/03. https://doi.org/10.1186/1472-6963-14-451.

12. Lehnert T, Heider D, Leicht $H$, Heinrich $\mathrm{S}$, Corrieri S, Luppa M, et al. Review: health care utilization and costs of elderly persons with multiple chronic conditions. Med Care Res Rev. 2011;68(4):387-420. Epub 2011/08/05. https:// doi.org/10.1177/1077558711399580.

13. Glynn LG, Valderas JM, Healy P, Burke E, Newell J, Gillespie P, et al. The prevalence of multimorbidity in primary care and its effect on health care utilization and cost. Fam Pract. 2011;28(5):516-23. Epub 2011/03/26. https:// doi.org/10.1093/fampra/cmr013.

14. Gress S, Baan CA, Calnan M, Dedeu T, Groenewegen P, Howson H, et al. Coordination and management of chronic conditions in Europe: the role of primary care--position paper of the European forum for primary care. Qual Prim Care. 2009;17(1):75-86 Epub 2009/03/14.

15. van Oostrom SH, Picavet HS, de Bruin SR, Stirbu I, Korevaar JC, Schellevis FG, et al. Multimorbidity of chronic diseases and health care utilization in general practice. BMC Fam Pract. 2014;15:61. Epub 2014/04/09. https://doi. org/10.1186/1471-2296-15-61.

16. Vogeli C, Shields AE, Lee TA, Gibson TB, Marder WD, Weiss KB, Blumenthal D. Multiple chronic conditions: prevalence, health consequences, and implications for quality, care management, and costs. J Gen Intern Med. 2007;22 (Suppl 3):391-5. https://doi.org/10.1007/s11606-007-0322-1.

17. World Health Organization. Global status report on noncommunicable diseases 2010. Description of the global burden of NCDs, their risk factors and determinants. Geneva: World Health Organization; 2011.

18. Travassos C, Martins M. Uma revisão sobre os conceitos de acesso e utilização de serviços de saúde. Cad saúde pública. 2004;20(supl.2):190-8.

19. Auqusto DK, Lima-Costa MF, Macinko J, Peixoto SV. Factors associated with the evaluation of quality of primary health care by older adults living in the Metropolitan Region of Belo Horizonte, Minas Gerais, Brazil, 2010. Epidemiol Serv Saude. 2019;28(1):e2018128. English, Portuguese. https://doi.org/10. 5123/S1679-49742019000100017.

20. Nunes BP, Soares MU, Wachs LS, Volz PM, Saes MO, Duro SMS, et al. Hospitalisation in older adults: association with multimorbidity, primary health care and private health plan. Rev Saude Publica. 2017:51:43. Epub 2017/05/12. https://doi.org/10.1590/s1518-8787.2017051006646.

21. Souza A, Faerstein E, Werneck GL. Multimorbidity and use of health services by individuals with restrictions on habitual activities: the Pró-Saúde Study. Cadernos de saude publica. 2019:35(11):e00155118.

22. Fortin M, Hudon C, Dubois MF, Almirall J, Lapointe L, Soubhi H. Comparative assessment of three different indices of multimorbidity for studies on health-related quality of life. Health Qual Life Outcomes. 2005;3: 74. Epub 2005/11/25. https://doi.org/10.1186/1477-7525-3-74

23. Valderas JM, Starfield B, Sibbald B, Salisbury C, Roland M. Defining comorbidity: implications for understanding health and health services. Ann Fam Med. 2009;7(4):357-63. https://doi.org/10.1370/afm.983.

24. Andersen RM. Revisiting the behavioral model and access to medical care: does it matter? J Health Soc Behav. 1995;36(1):1-10.

25. Malta DC, Bernal RTI, Lima MG, Araújo SSC, Silva MMAD, Freitas MIF, et al. Noncommunicable Diseases and the Use of Health Services: Analysis of the National Health Survey in Brazil. Revista de saude publica. 2017;51(suppl 1). https://doi.org/10.1590/S1518-8787.2017051000090.

26. Barata RB. Acesso e uso de serviços de saúde: considerações sobre os resultados da Pesquisa de Condições de Vida 2006. São Paulo perspect. 2008;22(2):19-29.

27. Mendoza-Sassi R, Béria JU. Utilización de los servicios de salud: una revisión sistemática sobre los factores relacionados. Cad saúde pública. 2001:17(4):819-32.

28. MacMahon S. Multimorbidity: a priority for global health research. London, UK: The Academy of Medical Sciences; 2018.

29. Viacava F, Bellido JG. Condições de saúde, acesso a serviços e fontes de pagamento, segundo inquéritos domiciliares. Ciência \& Saúde Coletiva. 2016;21:351-70 
30. Pilotto LM, Celeste RK. Tendências no uso de serviços de saúde médicos e odontológicos e a relação com nível educacional e posse de plano privado de saúde no Brasil, 1998-2013. Cadernos de Saúde Pública. 2018;34(4). https://doi.org/10.1590/0102-311×00052017.

31. Jankovic J, Mirkovic M, Jovic-Vranes A, Santric-Milicevic M, Terzic-Supic Z. Association between non-communicable disease multimorbidity and health care utilization in a middle-income country: population-based study. Public Health. 2018;155. https://doi.org/10.1016/j.puhe.2017.11.014.

32. Palladino R, Tayu Lee J, Ashworth M, Triassi M, Millett C. Associations Between Multimorbidity, Healthcare Utilisation and Health Status: Evidence From 16 European Countries. Age Ageing. 2016;45(3). https://doi.org/10. 1093/ageing/afw044

33. Pati S, Swain S, Hussain MA, Kadam S, Salisbury C. Prevalence, correlates, and outcomes of multimorbidity among patients attending primary care in Odisha, India. Ann Fam Med. 2015;13(5):446-50. Epub 2015/09/16. https:// doi.org/10.1370/afm.1843.

34. Hulka BS, Wheat JR. Patterns of utilization. The patient perspective. Med Care. 1985;23(5):438-60 Epub 1985/05/01.

35. Pinheiro RS, Viacava F, Travassos C. Brito AdS. Gender, morbidity, access and utilization of health services in Brazil. Ciênc saúde coletiva. 2002;7(4):687707. https://doi.org/10.1590/S1413-81232002000400007.

36. Travassos C, Viacava F, Pinheiro R, Brito A. Utilization of health care services in Brazil: gender, family characteristics, and social status. Rev Panam Salud Publica. 2002;11(5-6):365-73. https://doi.org/10.1590/S102049892002000500011 .

37. Violan C, Foguet-Boreu Q, Flores-Mateo G, Salisbury C, Blom J, Freitag M, et al. Prevalence, determinants and patterns of multimorbidity in primary care: a systematic review of observational studies. PLoS One. 2014;9(7): e102149. Epub 2014/07/23. https://doi.org/10.1371/journal.pone.0102149.

38. Wang HH, Wang JJ, Wong SY, Wong MC, Li FJ, Wang PX, et al. Epidemiology of multimorbidity in China and implications for the healthcare system: cross-sectional survey among 162,464 community household residents in southern China. BMC Med. 2014;12:188. https://doi. org/10.1186/s12916-014-0188-0

39. Diederichs C, Berger K, Bartels DB. The measurement of multiple chronic diseases-a systematic review on existing multimorbidity indices. J Gerontol A Biol Sci Med Sci. 2011;66(3):301-11. https://doi.org/10.1093/gerona/ glq208.

40. Mercer SW, Smith SM, Wyke S, O'Dowd T, Watt GC. Multimorbidity in primary care: developing the research agenda. Fam Pract. 2009;26:79-80.

\section{Publisher's Note}

Springer Nature remains neutral with regard to jurisdictional claims in published maps and institutional affiliations.

Ready to submit your research? Choose BMC and benefit from:

- fast, convenient online submission

- thorough peer review by experienced researchers in your field

- rapid publication on acceptance

- support for research data, including large and complex data types

- gold Open Access which fosters wider collaboration and increased citations

- maximum visibility for your research: over $100 \mathrm{M}$ website views per year

At $\mathrm{BMC}$, research is always in progress.

Learn more biomedcentral.com/submissions 